\title{
The unitary Fermi gas at finite temperature: momentum distribution and contact
}

\author{
Joaquín E. Drut* \\ Theoretical Division, Los Alamos National Laboratory, Los Alamos, NM 87545, USA. \\ E-mail: joaquindrut@gmail.com
}

Timo A. Lähde

Helsinki Institute of Physics and Department of Applied Physics, Aalto University, FI-00076

Aalto, Espoo, Finland

E-mail: talahdelgmail.com

\section{Timour Ten}

Department of Physics, University of Illinois, Chicago, IL 60607, USA.

Theoretical Division, Los Alamos National Laboratory, Los Alamos, NM 87545, USA.

E-mail: tten1@uic.edu

The Unitary Fermi Gas (UFG) is one of the most strongly interacting systems known to date, as it saturates the unitarity bound on the quantum mechanical scattering cross section. The UFG corresponds to a two-component Fermi gas in the limit of short interaction range and large scattering length, and is currently realized in ultracold-atom experiments via Feshbach resonances. While easy to define, the UFG poses a challenging quantum many-body problem, as it lacks any characteristic scale other than the density. As a consequence, accurate quantitative predictions of the thermodynamic properties of the UFG require Monte Carlo calculations. However, significant progress has also been made with purely analytical methods. Notably, in 2005 Tan derived a set of exact thermodynamic relations in which a universal quantity known as the "contact" $\mathrm{C}$ plays a crucial role. Recently, $\mathrm{C}$ has also been found to determine the prefactor of the high- frequency power-law decay of correlators as well as the right-hand-sides of shear- and bulk viscosity sum rules. The contact is therefore a central piece of information on the UFG in equilibrium as well as away from equilibrium. In this talk we describe some of the known aspects of Fermi gases at and around unitarity, show our latest Monte Carlo results for the contact at finite temperature, and summarize the open questions in the field, some of which we are starting to answer using large-scale Monte Carlo calculations by adapting methods from Lattice QCD.

The XXIX International Symposium on Lattice Field Theory - Lattice 2011

July 10-16, 2011

Squaw Valley, Lake Tahoe, California

\footnotetext{
${ }^{*}$ Speaker.
} 


\section{Introduction}

In recent years, our understanding of universality [1] in non-relativistic many-body quantum mechanics has increased dramatically. By universality we mean independence from the details of the interaction, in the same sense as in the context of second-order phase transitions, but in the absence of long-range correlations throughout the system (except potentially at special points in the phase diagram). Among the systems displaying this property, perhaps the most dramatic example is the so-called unitary Fermi gas (UFG). This system is a two-component Fermi gas tuned to the limit of vanishing interaction range $r_{0}$ and large s-wave scattering length $a_{s}$, and is termed "unitary" because it saturates the unitarity bound imposed on the scattering cross section by the unitarity of quantum mechanics. In short, the unitary gas is a resonant quantum mechanical many-body system.

A few years ago, the unitary limit was realized in metastable ultracold atomic clouds in various laboratories around the world (see Ref. [2] for a review of the experimental situation) and has been under intense scrutiny by the atomic, molecular and optical physics (AMO) community ever since [3]. Interest in the UFG transcends those areas, however, with a considerable amount of research being carried out within the nuclear physics community well before and after the first AMO experiments [4]. This, of course, is itself a manifestation of the universality of the UFG, as nuclear systems characteristically display short ranges and unnaturally large scattering lengths, although in a vastly different absolute scale than atomic clouds, the natural scale being in each case the Fermi momentum $k_{F}$.

More recently, the limit $0 \leftarrow k_{F} r_{0} \ll 1 \ll k_{F} a_{s} \rightarrow \infty$ has been shown to imply non-relativistic conformal invariance, as described in Ref. [5]. In turn, this results in a set of non-trivial relations between the system in homogeneous space and in a harmonic trap, as first shown in Ref. [6, 7]. In a separate line of research, short-distance correlations were shown by Tan $[8]$ and others $[9,10]$ to be completely encoded in a quantity $C$, which Tan called the "contact". Specifically, we may define the contact as

$$
C \equiv \lim _{k \rightarrow \infty} k^{4} n_{\sigma}(k)
$$

where $n_{\sigma}(k)$ is the momentum distribution for spin $\sigma$ expressed as a thermal average. This remarkable property stems in part from the short-range nature of the interaction, which implies that at resonance the many-body wavefunction is essentially that of a free gas, with the added boundary condition that it diverges as $1 / r$ when two coordinates are set to a short distance apart $r$ [11]. Following the work of Tan and others, the last couple of years have seen considerable activity extending the analysis of short-range correlations in many-body systems to systems away from unitarity as well as to different dimensions [12] and to a growing set of thermodynamic and even hydrodynamic quantities $[13,14]$. The latter, in particular, point to the fact that $C$ is relevant not only in equilibrium but also away from equilibrium (see Ref. [15] for a comprehensive review).

In spite of experimental advances and progress from the formal and analytic points of view, the UFG remains a challenging many-body problem. The reason for this is that, while resonant and therefore strongly interacting, the UFG has as few scales as a non-interacting Fermi gas. Enhanced symmetries aside, such a lack of scales implies lack of small parameters to perform an expansion, such that non-perturbative numerical methods are required. Indeed, while we know that Tan's contact plays a crucial role in the dynamics of the UFG, the only way to determine it accurately 
and reliably is by using numerical methods such as Quantum Monte Carlo (in any of its various incarnations, in particular on the lattice).

The work shown here represents the first attempt to determine the contact at finite temperature in a non-perturbative fashion. To this end, we have adapted methods from Lattice QCD, namely Hybrid Monte Carlo [16] and applied them to the calculation of the momentum distribution of the UFG. The next section outlines the main features of the algorithm and Sec. 3 shows our results and conclusions, which were first published in Ref. [18].

\section{Algorithm \& lattice formulation}

The lattice formulation we have used for this work follows closely that of Ref. [19], but differs in at least three notable aspects. Firstly, we determine the bare lattice coupling constant $g$ corresponding to the unitary regime by using Lüscher's formula [20] as in Ref. [21], without imposing a spherically symmetric cutoff. This procedure yields $g \simeq 5.144$ in the unitary limit. Secondly, we use the compact, continuous Hubbard-Stratonovich [22] transformation

$$
\exp \left(\tau g \hat{n}_{\uparrow i} \hat{n}_{\downarrow i}\right)=\frac{1}{2 \pi} \int_{-\pi}^{\pi} d \sigma_{i}\left[1+B \sin \left(\sigma_{i}\right) \hat{n}_{\uparrow i}\right]\left[1+B \sin \left(\sigma_{i}\right) \hat{n}_{\downarrow i}\right],
$$

where $\sigma_{i}$ (not to be confused with the spin projection) is the auxiliary field, with $B^{2} / 2 \equiv \exp (\tau g)-$ 1 , and $\tau$ denotes the lattice spacing in the imaginary time direction. We find that a time step $\tau \simeq 0.05$ is sufficiently small to render temporal discretization errors insignificant. The above representation (referred to as "Type 4" in Ref. [23]) was found to be superior with respect to acceptance rate, decorrelation and signal-to-noise properties than the more conventional unbounded and discrete forms [24]. Finally, the use of a continuous auxiliary field allows us to perform global updates using the Hybrid Monte Carlo (HMC) algorithm [16]. Our implementation of the HMC algorithm does not use pseudofermions but rather relies on a direct calculation of the fermion determinant in a purely spatial rather than spacetime formulation [17]. In addition, we use Fourier acceleration to propagate states in imaginary time. This enables global updates at all temperatures and lattice sizes, and scales approximately as $\sim V^{2} \log V$ (at fixed temperature) for moderate spatial lattice volumes $V$, to be contrasted with the $\sim V^{3}$ scaling of approaches based on local updates.

\section{Results and conclusions}

We have performed calculations at zero as well as finite temperature, in the former case using an approach similar to Ref. [23]. Our main results correspond to $40-50$ particles at $N_{x}=10$ and $70-80$ particles at $N_{x}=12$, in addition to limited data for $N_{x}=14$. In Fig. 1 (left panel), we show the momentum distribution $n(k)$ as a function of temperature $T / \varepsilon_{F}$. We have computed $n(k)$ by averaging over the angular directions on the lattice as well as over the imaginary-time slices. In this way, we find that $\sim 200$ uncorrelated auxiliary field samples for each datapoint gives excellent statistics for $n(k)$. Multiplying $n(k)$ by $k^{4}$, as plotted in the right panel of Fig. 1, we find a maximum at $k \simeq k_{F}$ and a leveling out at high momenta, with the asymptotic regime setting in at approximately $2 k_{F}$, at the lowest temperatures. There is no a priori reason for the asymptotic regime to set in at such low momenta; our work is the first to point out this fortunate situation. 
We study the temperature dependence of this "plateau", which allows us to determine the contact $C /\left(N k_{F}\right)$ as a function of temperature. The corresponding results are given in Fig. 2, together with a comparison with other theoretical predictions. Our results indicate that $n(k)$ follows the expected $\sim k^{-4}$ dependence accurately up to at least $k \simeq 4 k_{F}$, at which point the signal deteriorates, possibly due to lattice effects.
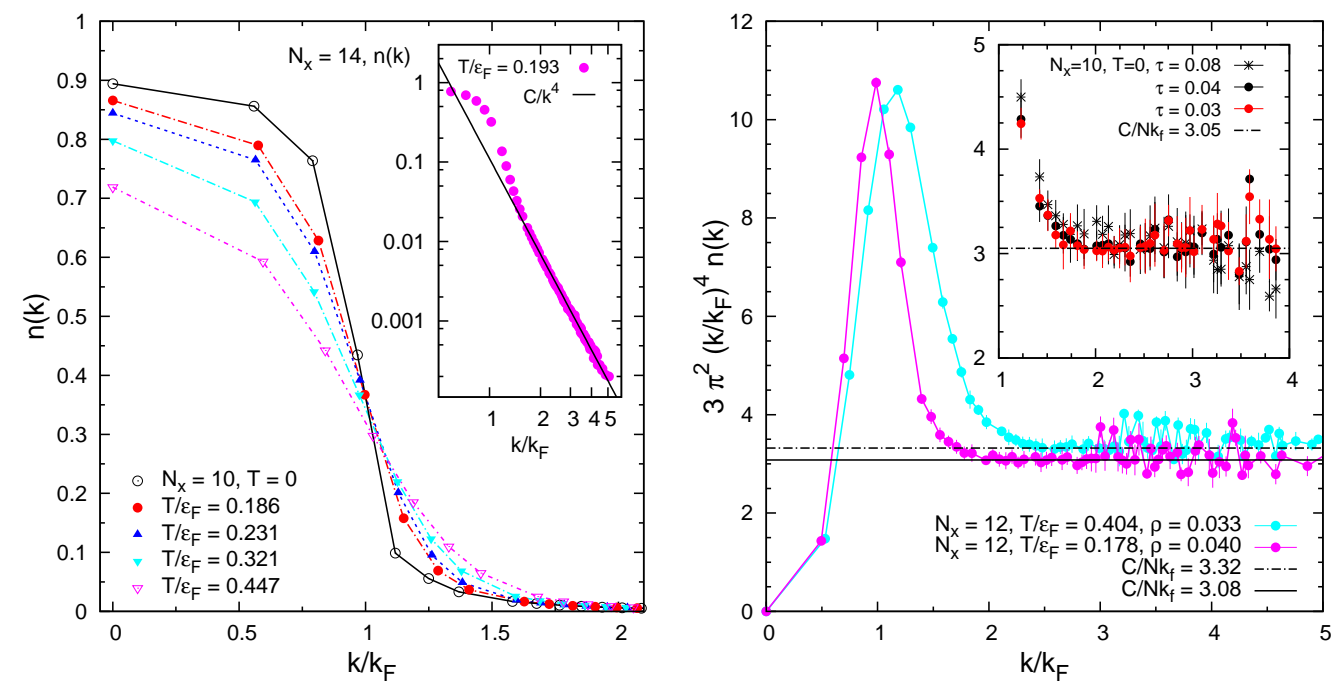

Figure 1: (Color online) Right panel: Momentum distribution $n(k)$ from $\mathrm{QMC}$ for $N_{x}=10$ as a function of $k / k_{F}$, for various temperatures ranging from zero to $T / \varepsilon_{F} \simeq 0.5$. The solid lines are intended to guide the eye, and the statistical errors are of the size of the symbols. Inset: $n(k)$ for $N_{x}=14$ in a log-log scale, showing the asymptotic $\sim k^{-4}$ behavior. Left panel: Plot of $3 \pi^{2}\left(k / k_{F}\right)^{4} n(k)$ for $N_{x}=12$ as a function of $k / k_{F}$ at $T / \varepsilon_{F}=0.178$ and 0.404 . The "plateaux" at large $k / k_{F}$ give the intensive dimensionless quantity $C /\left(N k_{F}\right)$. At low $T / \varepsilon_{F}$, the asymptotic region is reached at $k / k_{F} \simeq 2$. Inset: $N_{x}=10$ results at $T=0$ showing only slight dependence on the time step $\tau$.

Our results show that the contact $C$ grows with temperature well beyond the superfluid phase, which is suggestive of a peak $C_{\max } \simeq 3.4$ at $T / \varepsilon_{F} \simeq 0.4$. This scenario agrees qualitatively with Ref. [25], as well as Ref. [26]. Since $C$ measures the number of particle pairs (of both spins) whose separation is small, the appearance of a maximum indicates an enhancement in such short-range correlations. We find the scale at which the $k^{-4}$ law sets in (see Fig. 1) to be $k \simeq 2 k_{F}$ at finite $T / \varepsilon_{F}$ and somewhat lower for the ground state.

In summary, we have computed the momentum distribution $n(k)$ and the contact $C /\left(N k_{F}\right)$ for the UFG at zero and finite temperature, using a lattice formulation of the many-body problem, in conjunction with the HMC algorithm. Our results represent the first fully non-perturbative calculation of $n(k)$ free of uncontrolled approximations. We find that the contact at $T=0$ takes the value $\simeq 2.95 \pm 0.10$ and increases as a function of $T / \varepsilon_{F}$ in the low-and intermediate-temperature regimes that we have explored, which is consistent with the phonon-dominated scenario of Ref. [25]. Our results complement the calculations of Refs. [25, 26, 27, 28], and are suggestive of a maximum in $C /\left(N k_{F}\right)$ at $T / \varepsilon_{F} \simeq 0.4$, which agrees qualitatively with Ref. [26] but disagrees with Ref. [27]. While calculations at higher temperature $T / \varepsilon_{F} \sim 1$ are feasible, an improved understanding of the finite density effects is clearly called for. 


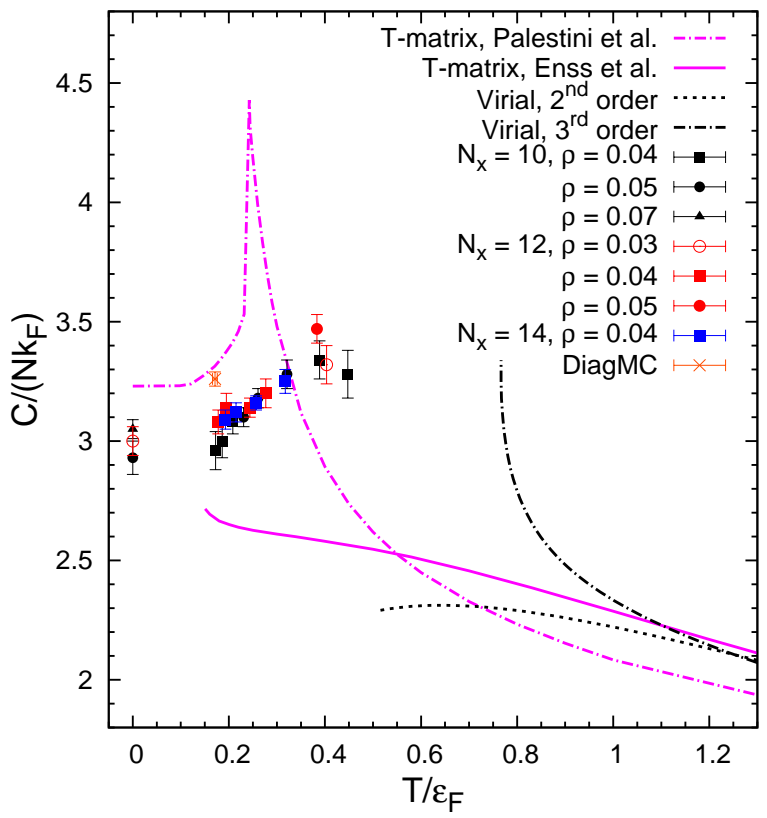

Figure 2: (Color online) Summary of QMC results for $C /\left(N k_{F}\right)$ as a function of $T / \varepsilon_{F}$, as determined from the large $k / k_{F}$ behavior of $n(k)$. The errorbars are dominated by systematics related to the residual fluctuations in the plateaux, as shown in the previous figure (right panel). Also shown are the t-matrix calculations of Ref. [26, 27], the virial expansion of Ref. [28] and the diagrammatic Monte Carlo result of Ref. [29].

\section{References}

[1] H. Heiselberg, Phys. Rev. A 63, 043606 (2001); T.-L. Ho, Phys. Rev. Lett. 92, 090402 (2004); E. Braaten, H.-W. Hammer, Phys. Rept. 428, 259 (2006).

[2] Ultracold Fermi Gases, Proceedings of the International School of Physics "Enrico Fermi", Course CLXIV, Varenna, 20-30 June 2006, edited by M. Inguscio, W. Ketterle, C. Salomon, IOS Press, Amsterdam (2008).

[3] S. Giorgini, L. P. Pitaevskii, S. Stringari, Rev. Mod. Phys. 80 (2008) 1215; I. Bloch, J. Dalibard, W. Zwerger, ibid. 80 (2008) 885.

[4] D. B. Kaplan, M. J. Savage, and M. B. Wise, Nucl.Phys. B534, 329 (1998); Phys.Lett. B424, 390 (1998); "The Many-Body Challenge Problem", formulated by G. F. Bertsch (1999), see e.g. Series on Advances in Quantum Many-Body Theory - Vol. 3, R. F. Bishop, K. A. Gernoth, N. R. Walet, Y. Xian (Eds.) (World Scientific, Singapore, 2000).

[5] Y. Nishida and D. T. Son, Phys. Rev. D 76, 086004 (2007).

[6] T. Mehen, Phys. Rev. A 78, 013614 (2008).

[7] F. Werner, Phys. Rev. A 78, 025601 (2008).

[8] S. Tan, Ann. Phys. 323, 2952 (2008); ibid. 323, 2971 (2008); ibid. 323, 2987 (2008).

[9] S. Zhang, A. J. Leggett, Phys. Rev. A 77, 033614 (2008). 
[10] E. Braaten, L. Platter, Phys. Rev. Lett. 100, 205301 (2008); E. Braaten, D. Kang, L. Platter, ibid. 104, 223004 (2010).

[11] F. Werner and Y. Castin, Phys. Rev. A 74, 053604 (2006).

[12] Y. Castin and F. Werner, arXiv:1103.2851. F. Werner and Y. Castin, arXiv:1001.0774.

[13] D. T. Son, E. G. Thompson, Phys. Rev. A 81, 063634 (2010).

[14] E. Taylor, M. Randeria, Phys. Rev. A 81, 053610 (2010).

[15] E. Braaten, arXiv:1008.2922.

[16] S. Duane et al., Phys. Lett. B 195, 216 (1987); S. A. Gottlieb et al., Phys. Rev. D 35, 2531 (1987).

[17] R. Blankenbecler, D. J. Scalapino, and R. L. Sugar, Phys. Rev. D 24, 2278 (1981).

[18] J. E. Drut, T. A. Lähde, T. Ten, Phys. Rev. Lett. 106, 205302 (2011).

[19] A. Bulgac, J. E. Drut, P. Magierski, Phys. Rev. Lett. 96, 090404 (2006); Phys. Rev. A 78, 023625 (2008).

[20] M. Lüscher, Commun. Math. Phys. 105, 153 (1986).

[21] D. Lee, T. Schäfer, Phys. Rev. C 73, 015201 (2006); Phys. Rev. C 73, 015202 (2006).

[22] R. L. Stratonovich, Sov. Phys. Dokl. 2, 416 (1958); J. Hubbard, Phys. Rev. Lett. 3, 77 (1959).

[23] D. Lee, Phys. Rev. C 78, 024001 (2008); Prog. Part. Nucl. Phys. 63, 117 (2009).

[24] J. E. Hirsch, Phys. Rev. B 28, 4059(R) (1983).

[25] Z. Yu, G. M. Bruun, G. Baym, Phys. Rev. A 80, 023615 (2009).

[26] F. Palestini et al., Phys. Rev. A 82, 021605(R) (2010).

[27] T. Enss, R. Haussmann, W. Zwerger, Ann. Phys. 326, 770 (2011).

[28] H. Hu, X.-J. Liu, P. Drummond, New J. Phys. 13, 035007 (2011).

[29] O. Goulko and M. Wingate, PoS (Lattice 2010), 187. 